\title{
Mercury Removal From Produced Water Using Subcritical Water Assisted 1-Etyl-3- Methylimidazolium Chloride lonic Liquid
}

\author{
Dr. Pradip Chandra Mandal ${ }^{1, \star}$ and Ahmad Fahmi Ridhuan bin Esa ${ }^{2}$ \\ ${ }^{1}$ Titas Gas T \& D Co. Ltd., 105 Kazi Nazrul Avenue, Kawran Bazar, Dhaka-1215, Bangladesh. \\ ${ }^{2}$ Universiti Teknologi PETRONAS, 32610 Bandar Seri Iskandar, Perak, Malaysia.
}

\begin{abstract}
Production of produced water from oil and gas industries are increasing day by day for enhancement of oil recovery. Insoluble mercury such as mercury sulfide and oxide, and dissolved mercury such as organomercury, ionic mercury and elementary mercury are present in produced water along with the other impurities. Mercury is not only hazardous to human health and the environment but could also attack process equipment components that have mercury reactive materials, leading to potential catastrophic failure to the plant. So, removing mercury from produced water is now a pressing issue for the environmental point of view. A lot of mercury separation techniques such as adsorption, precipitation etc. have been developed all over the world. But the traditional mercury removal processes are not suitable to remove mercury from produced water. The aim of this paper is to remove mercury from produced water using subcritical water assisted ionic liquid (IL) for the first time. In this study, 1-etyl-3methylimidazolium chloride, [EMIM][CI], IL was utilized. The potential experiments were conducted in a batch type extractor at temperatures of $130-180^{\circ} \mathrm{C}$, a pressure of $0.2 \mathrm{MPa}$ and treatment times of $0-10$ min. The concentration of mercury(II)ions in ILs were analyzed using UV-VIS spectrophotometer and IL was analyzed using FT-IR to study the structure of ILs. The results show that [EMIM][CI] IL has the ability to absorb mercury from produced water and almost $99.5 \%$ mercury was removed at a temperature of $180^{\circ} \mathrm{C}$, a pressure of $0.2 \mathrm{MPa}$ and a treatment time of $10 \mathrm{~min}$ by the action subcritical water assisted [EMIM][Cl]. In addition, mercury removal follows first order kinetics with an activation energy of $2.52 \mathrm{kcal}$ per mol and a pre-exponential factor of $1.093 \mathrm{~s}^{-1}$. Therefore, the subcritical water assisted ILs can be used to remove mercury from produced water of oil and gas industries.
\end{abstract}

Keywords: Mercury removal; Ionic liquid; Subcritical water; Mercury extraction; Extraction Kinetic

\section{Introduction}

Global awareness regarding mercury impact on environment has grown significantly over the past decade as mercury is not only hazardous to human health and the environment but also attack various parts of process equipment accelerating potential catastrophic failure of industries. In oil and gas industry, mercury presence in the natural gas, condensate, and crude oil. Mercury present in produced hydrocarbon can come to the environment by several avenues of emersions such as wastewater, solid waste streams and air emissions. Insoluble mercury such as mercury sulfide and oxide, and dissolved mercury such as organomercury, ionic mercury and elementary mercury are harmful for living beings. Generally, the rate of absorption of methylmercury in human body is higher than inorganic mercury. Methylmercury creates problem on central nervous system and inorganic mercury damages kidney. In oil and gas production perspective, the wastewater is defined as produced water. Separation of water is the initial process in production of both crude oil and natural gas. Separated water (produced water) is either discharge to the ocean or re-injected to the well to utilize it as enhance oil recovery (EOR). Produced water is the most enormous waste stream in oil and gas industry all over the world.

The removal of mercury ions from produced water is a prime distress for environmentalist. Mercury was found in the marine life at east coast of peninsular Malaysia. It is about median of $0.333 \mathrm{mg} / \mathrm{kg}$ mercury reading in marine life of east coast of Malaysia. While reading of marine life in Kuala Besar, Kelantan that is situated at the east coast of Peninsular Malaysia, gives $0.521 \mathrm{mg} / \mathrm{kg}$ mercury, second highest reading in Peninsular Malaysia[1]. Higher mercury contamination in Kuala Besar, Kelantan, has been observed due to anthropogenic activities in the South China Sea coastal water [2], oil wells activities in the confining areas, petroleum refineries, and oil tanker movements [3].Current technologies for aqueous mercury removal are precipitation, coagulation, reduction, membrane separation, ion exchange, and adsorption [4]. The primary methods of mercury removal in oil and gas industry are adsorption and precipitation. Adsorption method has the ability to remove $80 \%$ of heavy metal including mercury in produced water [5].

Ionic liquids (ILs) have shown the ability to remove both $\mathrm{Hg}^{2+}$ and $\mathrm{Hg}$ and more than $90 \%$ mercury has

*Corresponding author: Dr. Pradip C. Mandal CEng. MIChemE.

Tel.: +8801939921144, E-mail: pradipbd2002@yahoo.com 
been removed using this process. Mercury removal by this method has been well studied for mining industry and waste water. ILs have been used as replacements for conventional organic solvents in various separation processes as it is environmentally friendly [6]. ILs application opens a new way to make unusual solid-phase adsorbents. Several papers have been discussed various ILs, including imidazolium-based ILs, and phosphoniumbased ILs focusing on their ability toabsorborganic and inorganic compounds [7]. Unique physicochemical properties of ILs such as good thermal stability, and high removal efficiency, makes it is an efficient adsorbent for heavy metal ions [8, 9]. Large range of liquid state lead most of them in the liquid form in the ambient temperature, make them as room temperature ionic liquids (RTILs). They are claimed as thermally stable as they have high decomposition temperature. Decomposition temperatures of 1-Ethyl-3-methylimidazolium halides have been determined by DTG are $233^{\circ} \mathrm{C} 1$ Ethyl-3-methylimidazolium Chloride ([EMIM][CI]), $246 \stackrel{\circ}{\circ}$ 1-Ethyl-3-methylimidazolium Bromide, and $249 \stackrel{\circ}{\circ}$ 1-Ethyl-3-methylimidazolium lodide [10].

Changing in temperature able to give significant effect to the physicochemical properties of ionic liquids. Density, speed of sound, refractive index, and dynamic viscosity of ionic liquids changed when temperature changed [11]. Likewise, it is discovered that viscosity of $\left[\mathrm{CnMIM}_{[}\left[\mathrm{PF}_{6}\right]\right.$ decreased when the temperature of ionic liquid increased in the range $25-100^{\circ} \mathrm{C}$ [12]. These changing affected the percentage of efficiency in mercury ion removal by ILs. A complete mercury ion removal into the IL was achieved in the higher temperature. Additionally, faster mercury ion transfer also managed to be gained in the high temperature [13]. High viscosity of most alkyl-imidazolium reduces the rate of transfer of mercury ion into the ionic liquids. Practically, this behavior can be applied in the experiments to obtain higher efficiency of mercury ion removal.

Subcritical water is liquid water that has temperature and pressure above it's normal boiling point $\left(100^{\circ} \mathrm{C}\right.$, $0.1 \mathrm{MPa}$ ) and below critical point $\left(374^{\circ} \mathrm{C}, 22.1\right.$ $\mathrm{MPa}$ ). Water is maintained in the liquid state as the pressure of the water is constant. Subcritical water is literally stable due to liquid water is in equilibrium with vapour at the saturated vapour pressure. In this condition, liquid water behave in anomalous properties due to hydrogen bonding. Within the temperature range, the hydrogen bond is break and changing the properties of the water. Water becomes less polar, and behaves more like organic solvent. Organic matters solubility in water is increases by several orders, thus, water can be solvent, catalyst and reagent and can be used in extraction, chemical reactions and cleaning.
Subcritical water extraction is promising option of the traditional solvent extraction of heavy metal. Not only is the removal of heavy metal, also, the structure and morphology of the solvent retained [14]. Subcritical water usually produces desired physicochemical properties, and show suitable polarity. As mention before, water in high temperature behave as non-polar solvent. Solubility of water in organic compound increases as the temperature increase. Therefore, subcritical water can be employed as extraction medium. Akinlua et.al. used sub-critical water to extract trace metal from the petroleum source rock [15]. Besides, Hawthorne et. al. extract polycyclic aromatic hydrocarbons (PAH) by using subcritical water [16]. Utilization of subcritical water in the ionic liquid extraction can enhance the efficiency of the removal. Both ionic liquid and water is thermally stable in subcritical water condition. Thus, this property can be employed in the extraction of heavy metal from contaminated produced water. The aim of this study is to explore the capability of [EMIM][Cl] to remove mercury from produced water using subcritical water assisted ionic liquid (IL) for the first time.

\section{Methodology}

\subsection{Materials}

The $[\mathrm{EMIM}][\mathrm{Cl}] \mathrm{IL}$ was collected from lonic Liquid Center of Universiti Teknologi PETRONAS. Mercury (II) chloride was synthesized by reaction of elementary mercury and acid. The produced mercury (II) chloride was then used to produce produced water for the potential experiments.

\subsection{Experimental}

\subsubsection{Mercury (II) Chloride synthesis}

Exactly $50.2 \mathrm{~g}$ of elementary mercury was taken in a $500-\mathrm{mL}$ round bottom flask. Approximately $75 \mathrm{ml}$ of nitric acid, $\mathrm{HNO}_{3}$, was added slowly until all mercury reacted to form mercury (II) nitrate, $\mathrm{Hg}\left(\mathrm{NO}_{3}\right)_{2}$. Then the beaker with mixture was heated on a hot-plate until mercury (II) nitrate powder was formed. The dry mercury (II) nitrate powder was further heated for an additional two hours with occasional stirring. Under this condition, mercury nitrate was decomposed into a reddish, and yellowish white mercury (II) oxide. Mercury oxide was cooled down for a while. Then $60 \mathrm{~mL}$ of hydrochloric acid, $\mathrm{HCl}$, was mixed with the mercury (II) oxide powder and produced clear solution of mercury (II) chloride. Mercury (II) chloride was further heated to evaporate all water. 


\subsubsection{Produced water preparation}

Approximately $100 \mathrm{~mL}$ distilled water and $2 \mathrm{~g}$ of mercury (II) chloride was mixed in a $100-\mathrm{mL}$ beaker. The mixture was then stirred by magnetic stirrer for 5 min to produce produced water.

\subsubsection{Subcritical Water condition}

Ionic liquid was mixed with the produced water at a ratio of 1:10. The solution was then taken in a batch reactor. The batch extractor was placed in an oven for treatment times of $1-10 \mathrm{~min}$ at temperatures of 130 - $180^{\circ} \mathrm{C}$. After a specific temperature and treatment time, the extractor was removed from the oven, and then cooled at room temperature. Finally, ILs and water were separated and the samples were kept for analysis.

\subsection{Analysis}

Mercury (II) ion concentration in IL was analyzed using UV-VIS spectrophotometer. The separated IL samples were analyzed by FT-IR to understand the structure of IL. Mercury removal was calculated in order to identify the extent of removal of mercury in produced water using the following equation:

Mercury removal (\%) $=\frac{\mathrm{Hg}_{0}-\mathrm{Hg}_{\mathrm{t}}}{\mathrm{Hg}_{0}} \times 100$

where $H g_{0}$ is the initial mercury in loaded produced water and $\mathrm{Hg}_{t}$ is the mercury at treatment time, $t$.

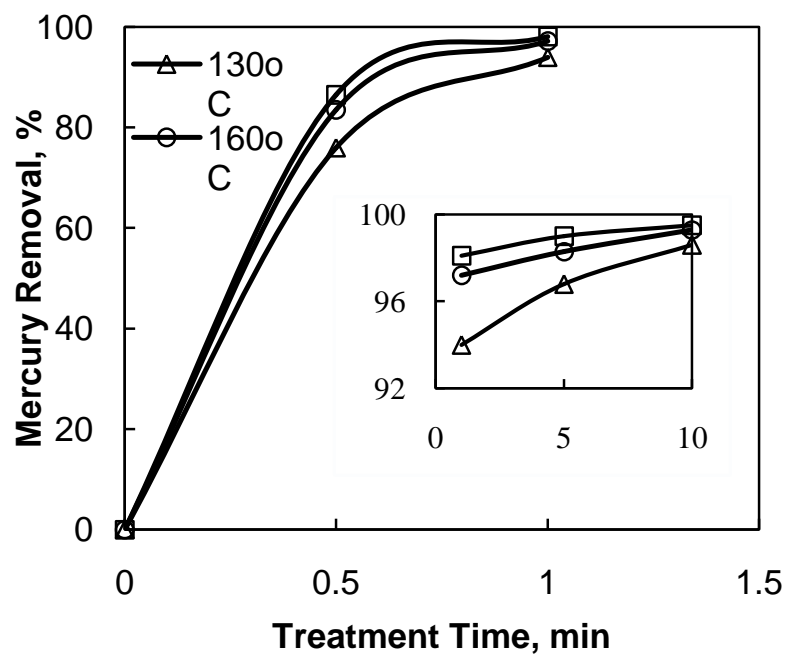

Fig. 1:Variation of mercury removal with respect of time

\section{Results and Discussion}

\subsection{Effect of Temperature}

To observe the capability of subcritical water assisted [EMIM][Cl] to remove mercury from mercury containing produced water, approximately twelve experiments were done at temperatures of $130-180^{\circ} \mathrm{C}$, a water partial pressure of $0.2 \mathrm{MPa}$, and treatment times of $0-10 \mathrm{~min}$. Fig. 1 shows the variation of mercury removal as a function of treatment time. It is observed that percentage of mercury removal was increasing with increasing treatment time and temperature at a specific water partial pressure. Approximately $99.50 \%$ mercury was removed at a temperature of $180^{\circ} \mathrm{C}$, a water partial pressure of $0.2 \mathrm{MPa}$, a treatment time of 10 min. The density of [EMIM][Cl] is high. With increasing temperature and treatment time, the density of [EMIM] $[\mathrm{Cl}]$ increases and mixed properly with the produced water and thus increase the solubility of mercury in [EMIM][Cl].

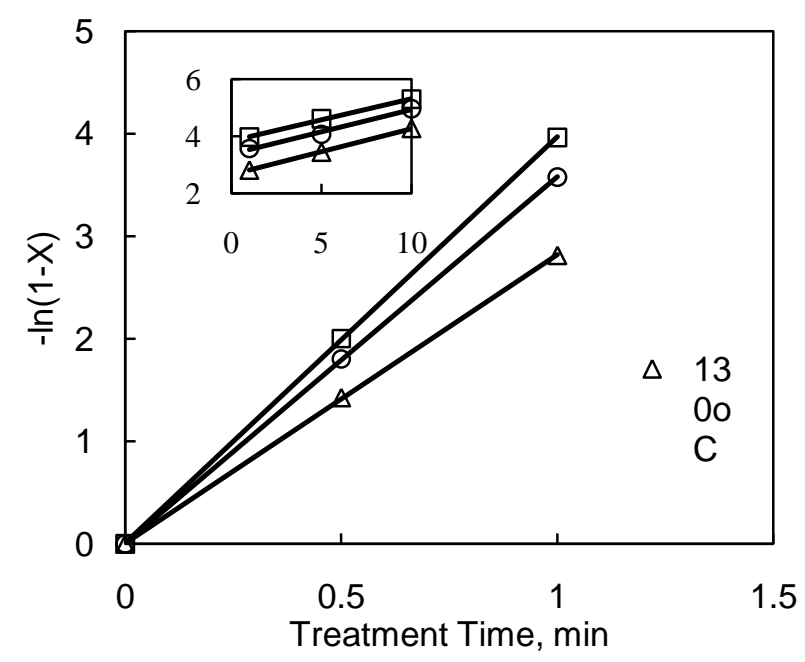

Fig. 2: A plot of $-\ln (1-X)$ vs. treatment time

\subsection{Mercury removal kinetics}

To discover the order of mercury extraction, a plot of $-\ln (1-X)$ where $X$ denotes fraction of mercury removal versus treatment time is constructed and displayed in Fig. 2. A straight that is obtained using least square method is almost passing through the origin. This behavior is the indication of first order kinetics with respect to mercury removal from produced water. In this study, the kinetics data were utilized and evaluated up to $98.1 \%$ mercury removal because the kinetics data were more fitted with the first order kinetics at this extent. Finally, an Arrhenius type temperature dependency mercury removal rate constant plot is prepared that is depicted in Fig. 3. Then, the activation energy and a pre-exponential factor were calculated by exploring this plot. The calculated values of activation energy and pre-exponential factor were $2.52 \mathrm{kcal} / \mathrm{mol}$ and $1.093 \mathrm{~s}^{-1}$ respectively. Thus, the rate constant equation for this process can be rewritten as follows:

$$
\mathrm{k}_{[\mathrm{EMIM}][\mathrm{Cl}]}=1.093 \mathrm{e}^{\frac{-2.52}{\mathrm{RT}}}
$$




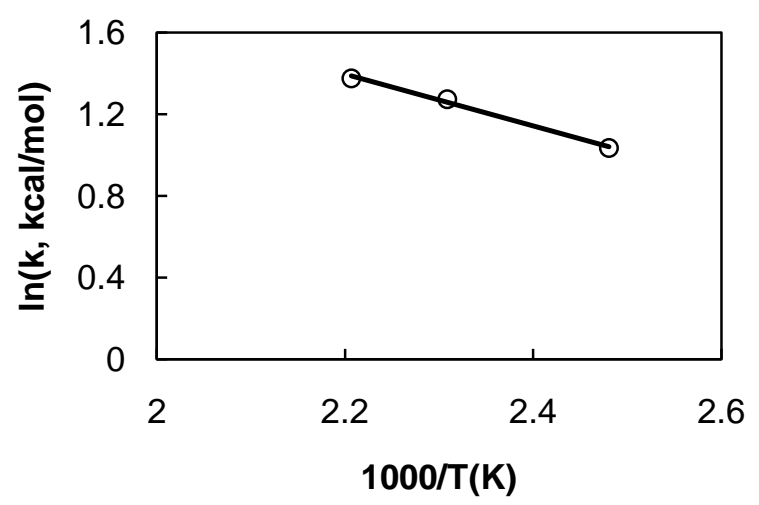

Fig. 3: Arrhenius plot

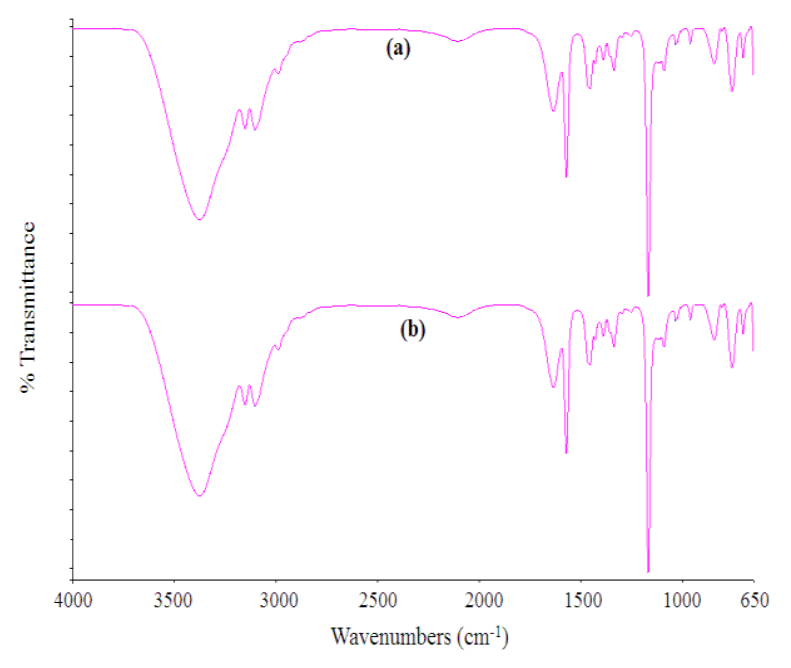

Fig. 4: FT-IR spectra of [EMIM][CI] (a) before treatment and (b) after treatment at a temperature of $180^{\circ} \mathrm{C}$ and a treatment time of $10 \mathrm{~min}$.

\subsection{FT-IR results}

The FT-IR spectra before and after the treatment is displayed in Fig.4. As alkyl methylimidazolium IL is used, peaks between $3000-1000 \mathrm{~cm}^{-1}$ is displayed. Peaks between that region show the structure of ionic liquids itself. It is seen that the characteristic peaks of [EMIM][CI] did not change or disappear. It is obvious that the treatment did not have significant effect on the nature of the IL. Therefore, in this process, $[\mathrm{EMIM}][\mathrm{Cl}]$ acted as an extractor of mercury. The statement of ion transfer as the partition in the ionic liquid could be strengthened by study IL structure. The IL shows that there is stretching in $850-100 \mathrm{~cm}^{-1}$ region. This stretching displayed that there is alkyl-halides ( $\mathrm{C}-\mathrm{Cl}$ stretch) in the IL. Thus, mercury could be partition of IL itself.

\section{CONCLUSION}

The extraction of mercury ion from produced water using subcritical water assisted [EMIM][Cl] $\mathrm{IL}$ is reported. The [EMIM][CI] IL was thermally stable up to the experimental condition. Mercury extraction efficiency increases as the temperature increases and maximum $99.50 \%$ mercury was removed from produced water by the action of sub-critical water assisted [EMIM][CI] IL with in a 10-min interval. The extraction kinetics follow first order kinetics with respect to mercury removal. Environmental benefits of ILs should be carefully considered. Not all ILs are nontoxic and safe. Therefore, the continuity of the development of real green ILs extraction should be carried on.

\section{REFERENCES}

1. N.I. Ahmad, M.F.M. Noh, W.R.W. Mohiyuddin, I. Ishak, W.N.F.W Azmi, Y. Vebo and H. Hairi, Environ. Sci. Pollut. Res.,22(5) (2015) 3672.

2. L. Liu, X. Xu, S. Yu, H. Cheng, Y. Hong, and X. Feng, Environ. Res., 131 (2014) 160.

3. B. Morton, and G. Blackmore, Mar. Pollut. Bull., 42(12) (2001) 1236.

4. J. Aguado, J.M. Arsuaga, and A. Arencibia, J. Hazard. Mater. 163(1) (2009) 213.

5. E. T. Igunnu, and G. Z. Chen, Int. J. LowCarbon Technol., 9(3) (2014) 157.

6. X. W. Wang, and S. Chen, Acta Chim. Sinica,72(11) (2014) 1147.

7. M. Regel-Rosocka, Sep. Purif. Tech. 66(1) (2009) 19.

8. J. Huang, M. Ye, Y. Qu, L. Chu, R. Chen, Q. $\mathrm{He}$, and D. Xu, J. Colloid Interface Sci., 385 (1) (2012) 137.

9. M. Kruk, and M. Jaroniec, Chem. Mater., 13 (10) (2001) 3169.

10. A. Efimova, L. Pfützner, and P. Schmidt, Thermochim. Acta, 604 (2015) 129.

11. A.B. Pereiro, H.I.M.Veiga, J.M.S.S. Esperança, A.J.Rodríguez, Chem. Thermodyn., 41 (2009) 1419.

12. J.G. Huddleston, A.E. Visser, W.M.Reichert, H.D. Willauer, G.A. Broker, and R.D.Rogers, GreenChem., 3(4) (2001) 156.

13. R. Germani, M.V. Mancini, G. Savellia, and N. Spreti, Tetrahedron Lett.,48(10)(2007) 1767.

14. E.Yabalak, and A. M.Gizir, J. Serb. Chem. Soc.78(7)(2013) 1013.

15. A. Akinlua, M. Roger, R. M. Smith, Talanta, 81 (2010) 1346.

16. S. B.Hawthorne, Y. Yang, and D. J. Miller, Anal. Chem., 66 (18)(1994) 2912. 